although only as a constituent of gliadin, the wheat protein. They found that blood glutamine levels rose in coeliac patients after oral dosage with gliadin. A. C. Frazer, C. A. C. Ross, and H. G. SAmmons (Birmingham) also reported the toxicity of gliadin in coeliac disease. Several communications on Castle's intrinsic factor were read. A. L. LATNER, R. J. MerRILls, and L. RaINE (Newcastle) reported the preparation of intrinsic factor in a highly purified state, apparently homogeneous on electrophoresis, ultracentrifugation, and chromatography. Intrinsic factor is a mucoprotein with a molecular weight of between 10,000 and 20,000 .

A. Bondi and Y. BIRK (Israel) found plant protein to yield large peptides resistant to the action of proteolytic enzymes. Animal protein did not contain such peptides and their results suggested to them a possible reason for the superior quality of animal protein in nutrition.

The section of chemical pharmacology produced a large variety of communications on numerous topics. C. $\mathrm{H}$. Gallagher, J. D. Judah, and K. R. ReEs (London) had investigated the mode of action of thioacetamide, a compound causing liver necrosis and regeneration, possibly carcinomatous. The hepatic necrosis was associated with a great increase in the amount of calcium in the liver cells, which might inhibit intracellular enzyme activity. Antihistamine drugs prevented the toxic effect of thioacetamide, and this and the calcium accumulation suggested to the authors a possible permeability effect.

\title{
Conclusion
}

It is difficult to sum up a scientific meeting covering as many topics as the Third International Congress of Biochemistry. One thing that did emerge, however, was the great amount of work now being devoted to the nucleoproteins and the prominence being given to such studies by those concerned with the cancer problem. Another feature of the congress was the steady progress apparent in the analysis of the chemical structure of proteins. Both this field and that of the chemistry of the cell nucleus reflect a trend towards precision of description and analysis and a tendency not to be contented with vague details of the structure of chemical compounds of biochemical interest. It is almost as if biochemistry were becoming more chemical and less bio'ogical.

In one respect the congress was disappointing. This was its failure to introduce any wholly new work on the achievement of respiratory enzymic activity and energy transformations in soluble as opposed to particulate systems. It seems reasonable to predict that by the time the Fourth International Congress is held in Vienna work of this nature will occupy a prominent place and will have led to notable advances.

\section{APPROVED NAMES}

Approved Names are devised or selected by the British Pharmacopoeia Commission. and the intention is that if any of the drugs to which these Approved Names are applied should eventually be described in the British Pharmacopoeia, the Approved Name should be its official title. The last list of approved names was published in the Journal of June 18, 1955 (p. 1473).

Approved Name

Aldosterone

Amiphenazole

Bemegride

Demecolcine

Glutethimide

Normethadone

Oxycinchophen

\section{Oiher Names}

$11 \beta$ : 21 - Dihydroxy - 3:20-dioxo-4pregnen-18-al. "Electrocortin"; "aldocorten."

2 : 4 - Diamino - 5 - phenylthiazole. "Daptazole."

$\beta$-Ethyl- $\beta$-methylglutarimide. " Megimide."

Deacetylmethylcolchicine. "Colcemid."

$\alpha$-Ethyl- $\alpha$-phenylglutarimide. " Doriden."

6-Dimethylamino-4 :4-diphenylhexan 3-one.

3-Hydroxy-2-phenylcinchonic acid.

\section{Correspondence}

Because of the present high cost of producing the Journal, and the great pressure on our space, correspondents are asked to keep their letters short.

\section{Treatment of Barbiturate Poisoning}

SiR,--Dr. A. Shulman's letter (Journal, July 30, p. 322) commenting on our paper (Journal, April 23, p. 1022) raises many interesting points.

Gastric Lavage.-There are clearly two possibilities to consider: the drug is either in the stomach (or the oesophagus) or it has passed through the pylorus. It is obviously futile to expect to recover by lavage that portion which has left the stomach. We do suggest, however, that it is always reasonable to perform gastric lavage in the hope of recovering some of the drug which may still be in the stomach. We should like to ask Dr. Shulman what he would do if a man said to him: "Doctor, ten minutes ago I swallowed 100 phenobarbitone tablets." It may be objected that the interval between taking the drug and starting treatment is rarely a matter of minutes but a few hours. At the time of the emergency, however, it is notoriously difficult to get reliable information on this point, and not infrequently the stated duration of narcosis is little more than an intelligent guess. Nor is time the only factor: the narcosis may be due to the immediate effects of sodium amytal, but who can say that there are not also a hundred tablets of phenobarbitone-the action of which has scarcely begun? Again, even when a long interval has elapsed before the start of treatment, it is by no means certain that barbiturate tablets or particles will not be present in the stomach or the oesophagus. In a case seen by one of us (S. A.) in a hospital abroad, tablets were recovered from the lower end of the oesophagus at post-mortem examination: for reasons which seemed excellent to the receiving physician, gastric lavage had not been carried out.

If, in fact, there is no barbiturate in the stomach, gastric lavage is valueless-but, if properly conducted, it is also harmless. Perhaps Dr. Shulman will reply that he does not deny the importance of emptying the stomach but that he prefers aspiration. We cannot agree that aspiration is the treatment of choice in the early stage of barbiturate poisoning; and in the later stages, if lavage is useless so also is aspiration. Dr. Shulman warns us against the danger of expediting the absorption of barbiturate by using large quantities of fluid during lavage. We emphasized the same point: "Not more than half a pint $(300 \mathrm{ml}$.) should be poured into the funnel at a time, because large volumes of fluid entering the stomach tend to drive the gastric contents through the pylorus." In support of his criticism of gastric lavage Dr. Shulman quotes Scandinavian workers who treated 100 serious cases of barbiturate poisoning by this method and found negligible quantities of the drug in the stomach washings. But these observations merely prove that gastric lavage was started too late - when nearly all the drug had passed through the pylorus; they do not invalidate the proposition that poisons lying in the stomach should be remeved. That this is the correct interpretation is supported by the fact that the cases were all regarded as "serious" when first seen-meaning, we suppose, that there was deep coma consistent with complete, or almost complete, absorption of the drug.

We would repeat, therefore. that competent gastric lavage should not be omitted: inevitably it will often be useless, but it should never be harmful, and occasionally it will be life-saving.

Sodium Bicarbonate.-Is often recommended for gastric lavage because it facilitates the removal of viscid mucus. We think it unwise, however, to use sodium bicarbonate in barbiturate poisoning, because insoluble preparations are likely to be converted to the soluble sodium salts and this hastens their absorption. Dr. Shulman's comments refer to the effects of sodium bicarbonate after absorption-an entirely different matter. We agree that if a mild alkalosis favours renal excretion of barbiturates administration of sodium bicarbonate may be justified. Two points, however, should be noted: (1) For the reasons stated above and because of the abnormal state of the alimentary canal in a deeply comatose patient, the sodium bicarbonate should not be given by mouth but by intravenous infusion. (2) In the later stages of coma, intravenous infusion of sodium bicarbonate solution would help to rectify the inevitable starvation ketosis.

Aperients.-Dr. Shulman refers to the possible harmful effects of aperients which would "serve as a source of irritation" in the atonic bowel. Our paper contained no recommendation of irritant purgatives. We mentioned magnesium sulphate: The 
function of this salt is to hinder the absorption of water from the lumen of the bowel and thus to delay the transfer of barbiturate to the blood stream. In the conditions under review-where an excess of water is available-magnesium sulphate is not an irritant.

Analeptics.-We welcome Dr. Shulman's authoritative comments on the merits of various analeptics available for the treatment of barbiturate intoxication. We entirely agree that "excessive doses" should not be used. He says that "there is still an inadequate individual index " for detecting such doses of analeptics. It seems to us highly improbable that the situation will ever be otherwise. Separate tests, such as pressure on the supraorbital nerves and recording the tendon reflexes, are admittedly unreliable. Nevertheless, by taking into account the whole clinical picture, the experienced doctor can nearly always recognize that the patient is regaining consciousness. We reject the suggestion of a fixed dose--implicit in his quoted statement referring to the use of amphetamine by other workers: "The suggested routine under these conditions [respiratory depression] is the intramuscular administration of amphetamine sulphate $(25 \mathrm{mg}$.)." It must be emphasized that the dose of an analeptic is that quantity which produces the desired result. The spacing of the dose fractions is a matter which can be decided only by the physician in charge of the individual patient. We note that it is Dr. Shulman's practice when using 2:4-diamino-5-phenylthiazole hydrochloride ("daptazole ") to give parenteral injections of $10 \mathrm{mg}$. at intervals of five minutes until satisfactory ventilation is obtained. Although, in this department, we are trying to determine the value of the newer analeptics, we are not yet prepared to express a firm preference for these as compared with amphetamine.

Finally, Dr. Shulman refers to the "excellent 'results" claimed by various authors who use analeptics sparingly but adopt "a thorough routine of medical management." It is well known that when a team of workers in an institution make a comprehensive study of a particular malady the results reflect their enthusiasm and attention to detail. In these circumstances it is not surprising to find that in every aspect of their work the standards are well above the average attained elsewhere. Such developments often provide models of therapeutic efficiency. However, they also create diffculties for an independent observer trying to assess the team's claims regarding the value of a new drug or the effect of withholding a particular therapeutic procedure. It is against this background that we have to examine new methods in the management of barbiturate poisoning. For obvious reasons mortality figures must be scrutinized with special care : their deficiencies can be made good only by resorting to controlled experiments; and designing such experiments within the limits of our code of ethics may well tax our ingenuity to the limit.-We are, etc.,

\section{T. J. THOMSON.}

Glasgow.

\section{Stanley Alstead.}

\section{Fluorohydrocortisone and Adrenal Hyperfunction}

SIR,-We have read with interest the report by Drs. C. L. Cope and R. J. Harrison (Journal, August 20, p. 457) on the effect of $9 \alpha$-fluorohydrocortisone in a case of Cushing's syndrome. We have carried out suppression tests of this type in four cases of Cushing's syndrome due to adrenal cortical hyperplasia; $10 \mathrm{mg}$. was given daily in one case for five days and in the others for three days. The effect on the adrenals was assessed by measuring the urinary excretion of 17-ketosteroids, 17-ketogenic steroids, and 17hydroxycorticoids. In one case all three showed a transient reduction; in one the 17 -ketogenic steroids and the 17 hydroxycorticoids fell but the 17-ketosteroids were unchanged, and in the third the 17-ketosteroids and the 17ketogenic steroids fell but the 17-hydroxycorticoids were not altered. In the fourth case, the most severe if assessed by hormone excretion, there was no definite response, although there was a gradual decline after the $9 \alpha$-fluorohydrocortisone was stopped. It is of interest that the patient who was given $9 \alpha$-fluorohydrocortisone for five days showed marked depression of steroid excretion on the first and second days and that after this the output increased.-We are, etc.,

London, W.1.

\section{"Veripaque"}

SiR,-Might I make some observations on statements recently published in the British Journal of Radiology ${ }^{1}$ concerning the above preparation? I had the privilege of carrying out at St. Mark's Hospital for Diseases of the Bowel a series of investigations using "veripaque" in 120 patients at apparently the same time as Drs. J. W. McLaren, King, and Copland were conducting their investigations elsewhere. I feel it would be in the interests of the profession to record our impressions concerning the action and results of this drug, because at St. Mark's we have not received a favourable impression of its action. One of my colleagues observed that the evacuation radiographs (to which Dr. McLaren attaches importance) were quite useless, with the mucosa stripped entirely of barium throughout the whole bowel in $75 \%$ or more of the cases.

There is nothing really new about this preparation, which, essentially, is simply a very powerful purgative, somewhat similar in action to, and as powerful as, phenolphthalein. In its original form it came to my notice as far back as 1952 under the name of "neodrast." It next made a reappearance in this country heralded as "colonopaque," and finally we now hear of its rebirth as "veripaque." I have good reason to believe that this drug has been discarded by most American opinions, and also rejected for use in enema work at St. Mark's. The paper published by Drs. McLaren, King, and Copland follows very closely the lines and details of that published by L. B. Schlegel."

The patients examined in my series followed one of three types of investigation: (1) patients treated with premedication by colonic lavage using sodium bicarbonate only in the wash-out solution, and then followed by the opaque enema combined with veripaque; (2) cases of severe constipation without any prior medication and using veripaque in the opaque enema only; (3) cases prepared by colonic lavage with veripaque in the washout but no veripaque in the opaque enema. The results were most disappointing, and were certainly not up to the standard of patients prepared by the routine technique of premedication with mild aperients followed by "double-flow" colonic lavage prior to the actual administration of the opaque enema.

Dr. McLaren and his co-workers think they have rediscovered the normal movements of the colon, using veripaque. The colon walls contain a mass of highly sensitive fine nerve termini forming numerous complicated delicate plexi, such as Auerbach's plexi, Meissner's plexi, etc., controlling the action of the longitudinal and circular musculature of the bowel walls. When veripaque is suddenly run into the colon, from the rectum to the caecum, these very sensitive nerve fibrils are violently stimulated by this powerful purgative. In some of my patients the reaction was almost volcanic. They experienced great pain, broke into beads of perspiration; and one said she felt she "would like to die." How could such a violent stimulation be recorded as presenting the movements of a normal bowel ? And no wonder the caecum can be seen to assume a violent and unusual contraction and curl up upon itself, being the only unattached and free end of the colon. If a pin is stuck into a worm near one of its ends, it also sharply curls its end upon itself. The movements of a colon violently stimulated by veripaque are far from normal, and indeed rather resemble those of a patient who has had an overdose of strychnine injected. For many years at St. Mark's we in the $x$-ray department have accepted that the primary stimulation in the colon starts by contraction at approximately the recto-pelvic junction. The early-morning cup of tea usually arouses stimulation in this area, gentle at first, then gradually increasing in power of contraction. Soon local gas collection may occur in the rectum and subsequently the desire to defaecate comes on. With the rectum filled, the pressure contraction from the recto-pelvic area empties these sections of the bowel at stool. Should the act of defaecation not be permitted at the moment of call, the contracting mechanism in the recto-pelvic junction eventually relaxes. Some patients can actually feel this relaxation, with the bowel contents regurgitating back into the pelvic section with its highly mobile and astonishing telescopic muscle expanding and making way for the return of faecal material, until another peristaltic wave returns later on. If, however, the call to stool is allowed to function and the act of defaecation carried out, resulting in the emptying of the rectum and sigmoid, then some short time later the faecal collection of the descending section moves down, to be later followed by another sectional movement of the transverse section, and subsequently the 